\title{
ESTIMATE OF COMPONENTS RELIABILITY AND MAINTENANCE STRATEGIES IMPACT ON TRAINS DELAY
}

\author{
Giovanni Dazzi and Stefano Savio \\ Electrical Engineering Department - University of Genova \\ Via all'Opera Pia 11a, Genova 16145, Italy \\ E-mail: stefano@die.unige.it
}

\author{
Pierluigi Firpo \\ D’Appolonia S.p.A. \\ Via San Nazaro 19, Genova 16145, Italy \\ E-mail: pierluigi.firpo@dappolonia.it
}

\section{KEYWORDS \\ Reliability, Maintenance, Train delay, Monte Carlo}

\begin{abstract}
In this paper the authors present a procedure for estimating the impact of design choices, dealing with subsystems architecture, basic components reliability performances or maintenance policies, on the service quality provided by a railway or metro system. The procedure is based on the use of an innovative modular simulator, developed by the authors. Through a Monte Carlo approach and a suitable post-processing of the results of the simulated samples, the tool provides the user with a set of indicators for estimating the service quality and the effectiveness of different traffic and fleet management policies, as well as maintenance strategies, in both rated and degraded operating conditions.
\end{abstract}

\section{INTRODUCTION}

In order to evaluate service quality, that is to say the measure of the level of satisfaction of the users, the parameter Service Dependability may be introduced (Calabria et al. 1993). Service Dependability $(S D)$ is usually defined as the probability that a train, during a generic travel, collects a delay $d$ not greater than an allowable quantity $\delta$. Such a parameter is related to service quality as it takes into account the major request of the passenger, which every day utilizes railway and metro transit systems: for the user, in fact, only short and rare train delays are acceptable. The first step to evaluate $S D$ is to identify all the failure modes of the system components, estimate the probabilistic occurrence of such events and correlate failure modes impact with both the locos allowed performances and the mutual interactions among the vehicles.

Some of the authors have already analysed the problem from an analytical point of view, demonstrating that such approach is possible in very simple situations only. For instance, it is possible to evaluate the probabilistic impact of loco electrical drive failures on the running time of the vehicle (Cosulich et al. 1996), considering it as a stand alone unit (no interactions with other vehicles) and only in the case heavy simplifications are introduced.

If all the other railway or metro subsystems (Electrical substations, overhead line, signalling system, ...) are taken into account, as well as their relevant failure modes, the mentioned approach becomes impossible and the only solution is the adoption of simulation tools (Bozzo et al. 2003), where the prediction of the RAM (Reliability, Availability, Maintainability) performances of each subsystem plays a fundamental role. In such a context RAM prediction can be defined as a simulation process to identify the occurrence of failure events and repair actions for each macro-component (subsystem) and analyse the effects of both failures and (corrective and preventive) maintenance operations on the performances of the transportation system under investigation, as far as traffic behaviour is concerned.

Starting from rated conditions, where all the subsystems are correctly operating and departure and arrival times are the scheduled ones, the proposed tool (Dazzi et al. 2006) generates by Monte Carlo method the Times To Failure (and Times To Repair) for each basic component and performs a detailed analysis of the traffic evolution whenever a failure event occurs, identifying the delay collected by the trains, till rated conditions are reached again (taking into account repair actions as well). The result of such analysis represents a sample of the delays population, and the collection of a suitable number of those samples (simulations), integrated with a suitable statistical post processing, allows the designer to estimate the actual quality level of the system, detect possible bottlenecks and define the required corrective actions to be implemented, if any. Moreover, it is worth mentioning that other $S D$ indicators, different from the one previously defined, can be statistically estimated by the tool utilising the same simulation procedure (for instance, an $S D$ indicator related to the number of lost runs in a metro application).

\section{THE INNOVATIVE SIMULATION TOOL}

When the railway or metro system is operating in rated conditions, a failure event in one (or more) of the technological macro-components (subsystems) may occur. This failure may imply a partial or total loss of the ability of the macro-component in completing its mission and, conversely, a decrease in the quality of the service provided by the entire transport system (trains delay or loss of runs).

The proposed tool basically consists of two different simulators, duly integrated and interacting:

$>$ a traffic simulator;

$>$ a failure simulator.

The former analyses the traffic behaviour taking into 
account all the constraints deriving from the rated or degraded performances of the different macrocomponents, while the latter, based on the Monte Carlo method, generates the failure occurrences for those macro-components and the "rules" that apply when each different failure is injected into the system.

The design has been carried out by the authors in order to achieve an optimal compromise between modelling accuracy and computing time, and to fulfil two main requirements:

$>$ flexibility, related to both the input data and output results, and dealing with the possibility to analyse systems with different layouts, characteristics of rolling stocks, schedules, fleet management and maintenance policies, and to estimate different user defined $S D$ indicators;

$>$ modularity, dealing with the possibility to implement a new model for a subsystem by just adding the relevant module, without any further modifications (each module is a "black box" interacting with the others through a suitable interface only).

The traffic simulator can perform the analysis of the trains dynamic movement over a layout characterized by:

$>$ any number of passengers stations and freight terminals;

$>$ any number of maintenance depots or marshalling yards;

$>$ any gradient profile;

$>$ any number and configuration of Electrical substations (ESSs);

$>$ fixed or moving block signalling systems.

Each train is characterised by its own length, weight, rolling and aerodynamic drag characteristics, and different locos can be taken into account through the definition of their own weight and their relevant traction effort and deceleration curves. At last, different traffic and fleet management policies, as well as maintenance strategies, can be defined and implemented. As far as the failure simulator is concerned, the Monte Carlo method has been chosen for the generation of the Times To Failure (TTFs) and of the Times To Repair (TTRs) of the different subsystems components over a predefined time horizon, once known their reliability and maintainability characteristics. The Time To Failure and the Time To Repair are random variables characterised by their relevant Probability Density Functions (PDFs). The knowledge of such PDFs is mandatory for performing the RAM analysis of the system, as the Cumulative Distribution Function (CDF) $F(t)$ of a random variable can be computed starting from the relevant $\operatorname{PDF} f(t)$ as it follows:

$$
F\left(t_{0}\right)=\int_{0}^{t_{0}} f(t) d t
$$

being $F\left(t_{0}\right)$ the probability that the stochastic variable $t$ is not greater than a given value $t_{0}$, being $t \in(0,+\infty)$. For each value of the random variable $t$ in $(0,+\infty), F(t)$ assumes values uniformly distributed in $(0,1)$. So, samples of the random variable $t$ distributed according to $F(t)$ can be obtained generating a random number in $(0,1)$ and subsequently inverting $F(t)$.

For instance, if the random variable $t$ is exponentially distributed, the relevant PDF $f(t)$ can be expressed as:

$$
f(t)= \begin{cases}\lambda e^{-\lambda t} & t \geq 0 \\ 0 & t<0\end{cases}
$$

where $\lambda$ is a constant, and the Cumulative Distribution Function $F(t)$ is:

$$
F(t)=1-e^{-\lambda t}
$$

If the TTF of a component is characterised by such a distribution, a sample can be generated solving the following equation:

$$
\mathrm{TTF}=\frac{\ln (1-F)}{-\lambda}
$$

where $\lambda$ is the reciprocal of the component MTTF and $F$ is, for any sample to be generated, a random number in $(0,1)$ get by Monte Carlo method (Gedam et al. 2000, Bozzo et al. 2003). The Monte Carlo based approach has been adopted by the authors due to its flexibility, as it allows to carry out the failure analysis for components whose TTF (or TTR) is characterised by any distribution (exponential, Weibull, lognormal, ...).

According to the reliability connection of the basic components of the subsystem (series, parallel, stand-by, load sharing, k-out-of-n, ...) and the level of detail chosen for modelling its real structure, a performance constraint at the subsystem level is then associated to each failure occurrence to drive the traffic simulator.

\section{TRAIN ELECTRICAL DRIVE MODEL}

The case study analysed by the authors is related to a railway system adopting a multivoltage vehicle designed for the Italian High Speed Railway System (Bertini et al. 1990, Bogliani et al. 1992). The vehicle was designed in order to operate with all the power supply systems usually adopted in Europe: $3 \mathrm{kV}$ DC, $1.5 \mathrm{kV} \mathrm{DC}, 25 \mathrm{kV} 50 \mathrm{~Hz}, 15 \mathrm{kV} 162 / 3 \mathrm{~Hz}$.

The overall structure of the vehicle consists of three equivalent modules ( $\mathrm{A}, \mathrm{B}$ and $\mathrm{C}$ ), each composed of two motoring coaches (two motorised boogies) and one towed coach. The propulsion system of each module is made up of an asynchronous motor drive, with four induction motors, $550 \mathrm{~kW}$ rated power each.

The propulsion chain, where power electronics plays a fundamental role, is modular and can be configured as a function of the power supply system. In Figure 1 the traction chain configuration analysed in this paper and related to the case of the $25 \mathrm{kV} 50 \mathrm{~Hz}$ feeding system is shown: this scheme presents a transformer with two secondary windings $\left(\mathrm{N}_{\mathrm{p}}, \mathrm{N}_{\mathrm{s} 1}, \mathrm{~N}_{\mathrm{s} 2}\right)$, two decoupling 
inductors $\left(\mathrm{L}_{11} / \mathrm{L}_{12}, \mathrm{~L}_{21} / \mathrm{L}_{22}\right)$ and two four quadrant converters which feed the DC-link, made up of the 100 $\mathrm{Hz}$ resonant filter $\left(\mathrm{L}_{1 \mathrm{f}} / \mathrm{L}_{2} \mathrm{f}, \mathrm{C}_{1 \mathrm{f}}-\mathrm{C}_{2} \mathrm{f}\right)$ and the capacitor $\mathrm{C}_{\mathrm{i}}$, which stabilises the inverter input voltage; the inverter feeds four paralleled induction motors.

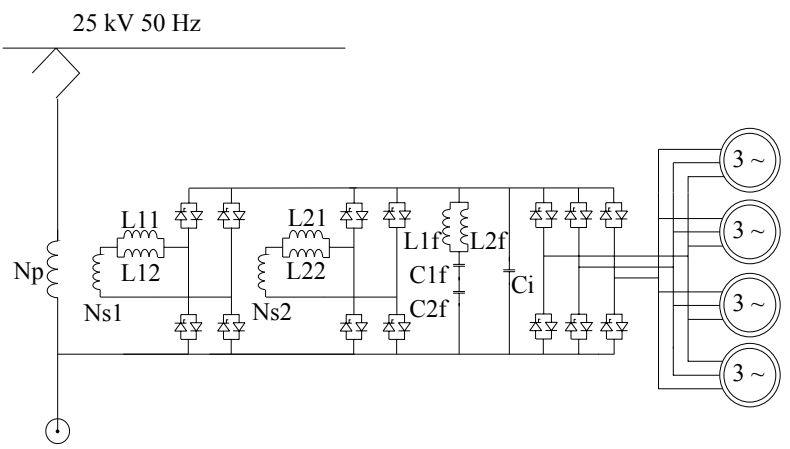

Figure 1 - Traction drive (25 kV $50 \mathrm{~Hz}$ feeding system)

The vehicle Reliability Block Diagram (RBD) is shown in Figure 2: the RBD consists of three identical reliability macro-blocks, in parallel configuration. Each macro-block models the traction drive shown in Figure 1, together with the boogies, each composed of two bearings and one reduction gear. It may be decomposed into three blocks (transformer, filters and inverter) in series configuration; two blocks, describing the two four quadrant converters, in parallel configuration; four blocks, representing the induction motors, two by two in series configuration with the related boogie, and then paralleled.
The configuration adopted for the induction motors takes into account that, whenever one motor of a coach fails, the other motor of the same coach is switched off for mechanical reasons. Each module is assumed to be able to provide three different power levels: for instance, the traction module is able to supply full power if all its components are correctly operating; it is able to supply half power if one four quadrant converter is out of order or one induction motor fails, while no traction power is available if both the four quadrant converters or one of the transformer, filters or inverter blocks are out of order. Zero power is provided by the module also in the case two motors (one for each coach) are out of order. When one boogie fails, the vehicle is able to complete the trip at reduced speed, whose maximum value cannot exceed $80 \mathrm{~km} / \mathrm{h}$. It has been also assumed that no maintenance action is performed during the mission time.

Table 1 lists the Mean Time To Failure (MTTF) values of the system blocks depicted in Figure 2; those data are obtained from manufacturers experience and from international standard (IEEE Std 493 1990, MILHDBK-217 1991). For electrical and electronic components an exponential distribution has been assumed, while for the boogies a normal distribution has been supposed, with a standard deviation equal to $300 \mathrm{~h}$. This case study has been presented by the authors to show the potentialities of the procedure and how it can be easily customised to solve different problems: it represents a complex system which can be maintained at the depot level only and whose performances (running times) vary as a function of the failed components.

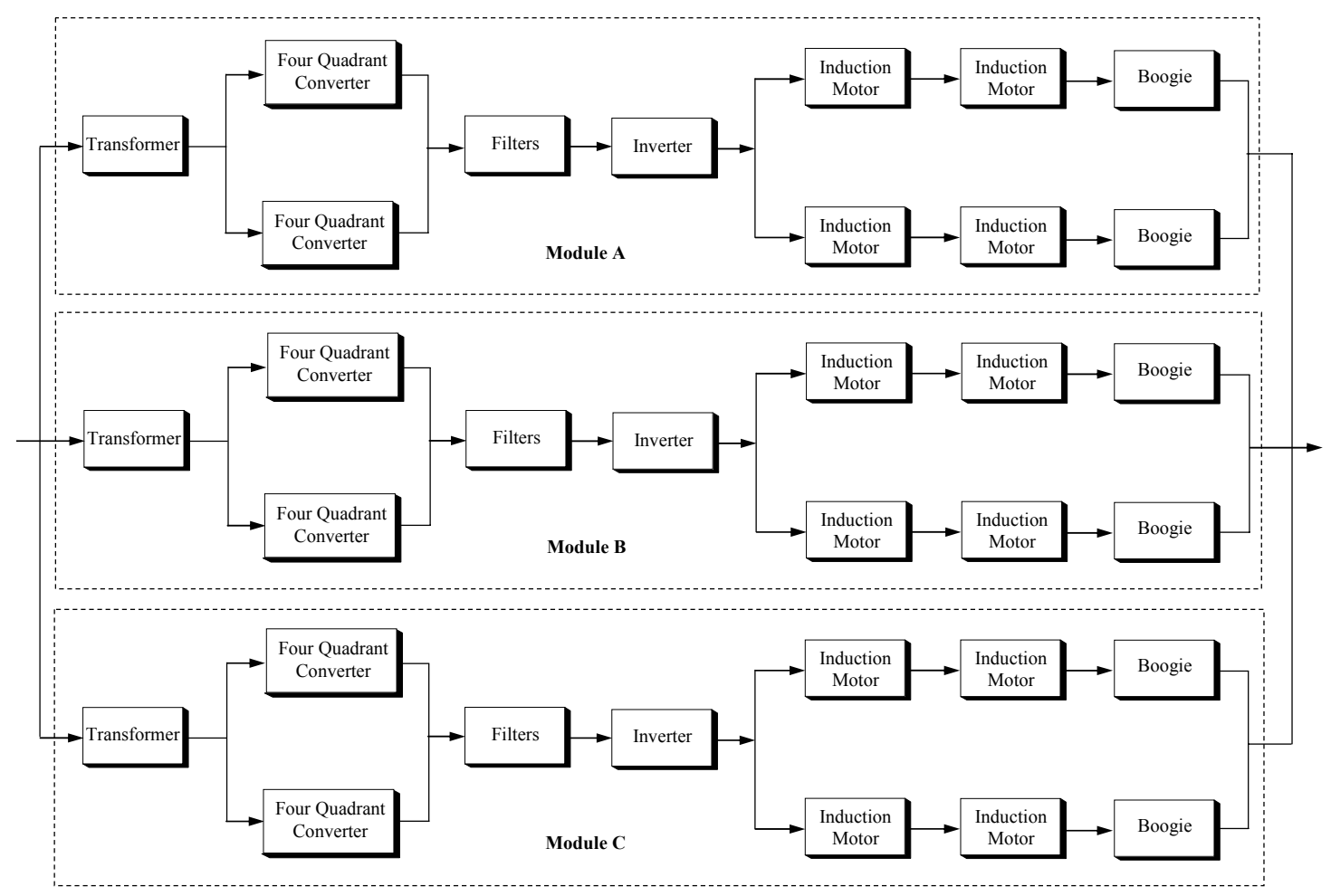

Figure 2 - Vehicle Reliability Block Diagram 
Table 1: System blocks MTTF

\begin{tabular}{|c|c|}
\hline Block & MTTF [h] \\
\hline Transformer & 450000 \\
\hline Four Quadrant Converter & 35700 \\
\hline Filters & 2500000 \\
\hline Inverter & 26300 \\
\hline Induction motor & 125000 \\
\hline Boogie & 1100 \\
\hline
\end{tabular}

The reliability model previously defined has been implemented into the tool in order to provide the traction power availability of each running train during the simulation. Thanks to such an information, when a failure occurs, the traffic simulator computes the available traction effort for all the vehicles on the track and solves the relevant dynamic motion equations. At last it has been supposed to have always available a constant deceleration both in rated and degraded conditions.

Should an immobilising failure occur (maximum speed equal to zero), the running time is computed as the passenger travel total time: the time from the departure till the stop along the track is summed to the time required by a spare unit, starting from the nearest station, to reach and take on board the passengers and to complete the travel. In this case no failure is supposed to occur in the spare unit.

\section{SIMULATION RESULTS}

In this chapter the authors present the results dealing with the simulation of a High Speed railway system, whose track layout is depicted in Figure 3 together with the related slope profile.

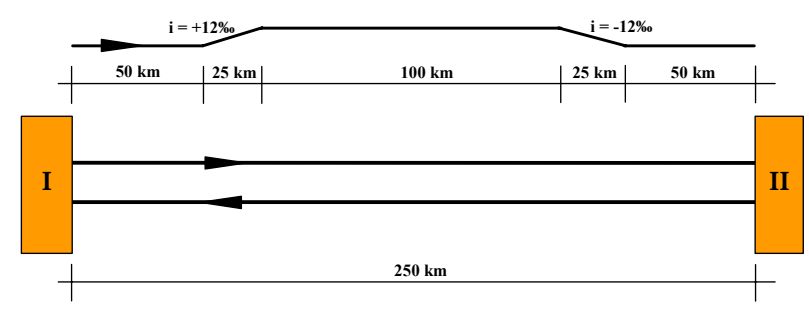

Figure 3 - Track layout and slope profile

In the simulated railway system, a double track line connects two passenger stations. Station I is $250 \mathrm{~km}$ far from Station II and the track is flat everywhere but in two sections, each $25 \mathrm{~km}$ long, characterised by a $\pm 12 \%$ o slope (positive slope for trains leaving a station). The two sections are placed $50 \mathrm{~km}$ and $175 \mathrm{~km}$ far from the departure station respectively. The maximum allowed cruising speed is $300 \mathrm{~km} / \mathrm{h}$, but a speed restriction $(80$ $\mathrm{km} / \mathrm{h}$ ) is present when the train approaches the arrival station. The railway line is equipped with a moving block signalling system and a maintenance depot is present at each passenger station.

All the running trains are characterised by the reliability model discussed in the previous chapter, and their running profile in rated operating conditions is depicted in Figure 4. It is worth mentioning that the speed decrease occurring after the start-up phase is just due to the track slope, whose value does not allow the train to maintain the maximum speed even if the full traction power is available. Travelling time equals about 58 minutes and, according to the adopted headway (15 minutes) and timetable, eight trains are contemporarily present over the track. At last, eight spare units are available at the beginning of the simulation in the depot of each passenger station.

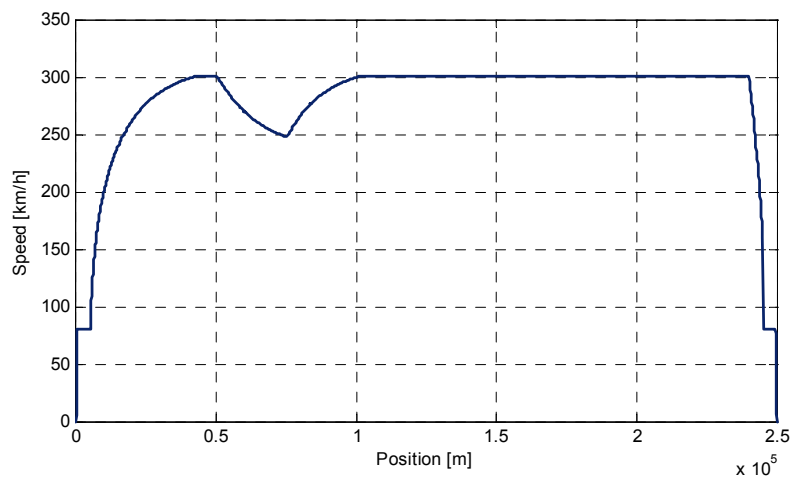

Figure 4 - Running profile in rated conditions

The authors has applied the proposed procedure to the case study for estimating the impact on service quality of the train drive components reliability and maintenance strategy.

In particular, the procedure has been utilised for computing conventional measures, such as the mean delay of the train, and has been also used for estimating of the Service Dependability $(S D)$, which can be formalised by the following relationship, where $d$ is the stochastic variable "delay" and $\delta$ an allowable quantity:

$$
S D=\operatorname{Pr}\{d \leq \delta\}
$$

The authors have analysed about 73000 samples (observed runs) by performing six replications of a simulation carried out over a time horizon of 10000 operating hours.

The results depicted in the following refer to two different case studies (Case A and Case B). In Case A it has been supposed to adopt a maintenance strategy which calls for a preventive maintenance action on the mechanical equipment of the loco (boogie) every 1000 operating hours. Case B refers to a situation where preventive maintenance actions are carried out every 2000 operating hours. For both case studies, the effect of a preventive maintenance action is an $80 \%$ renewal of the mechanical equipment.

Figure 5 and Figure 6 show the delay histograms for Case A and Case B respectively. For a better readability the authors have preferred to show in Figure 5 and Figure 6 delayed trains only and to associate each delay interval to the number of the delayed trains, computed as a percentage with respect to the overall number of delayed trains. 


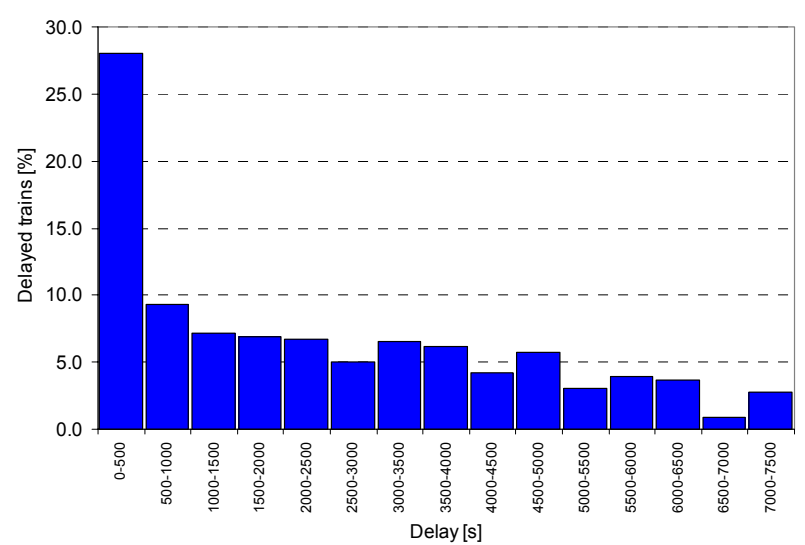

Figure 5 - Delay histogram (Case A)

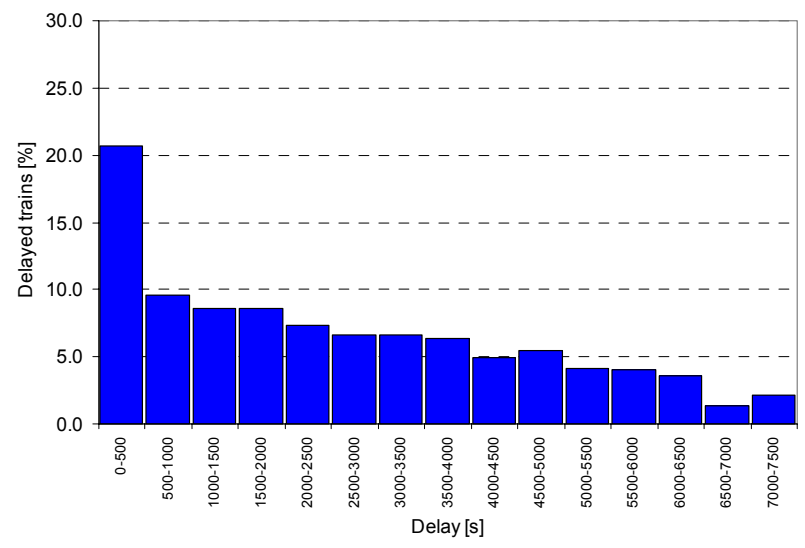

Figure 6 - Delay histogram (Case B)

Table 2 and Table 3 present the results of the measures performed on the observed samples: the mean value of the distributions is shown together with the estimated standard deviation $\sigma_{\mathrm{m}}$ of the sample means (Case A and Case $\mathrm{B}$ respectively).

Table 2: Post processing measures (Case A)

\begin{tabular}{|c|c|c|}
\hline \multirow{2}{*}{ Measure } & \multicolumn{2}{|c|}{ Value [s] } \\
& Delayed trains & All trains* \\
\hline Mean delay & 2378 & 25 \\
\hline Std deviation $\sigma_{\mathrm{m}}$ & 77 & 1.2 \\
\hline $90 \%$ SC interval & $(2251,2505)$ & $(23,27)$ \\
\hline 95\% SC interval & $(2224,2532)$ & $(22,28)$ \\
\hline * rounded values
\end{tabular}

Table 3: Post processing measures (Case B)

\begin{tabular}{|c|c|c|}
\hline \multirow{2}{*}{ Measure } & \multicolumn{2}{|c|}{ Value [s] } \\
& Delayed trains & All trains* \\
\hline Mean delay & 2552 & 36 \\
\hline Std deviation $\sigma_{\mathrm{m}}$ & 63 & 1.4 \\
\hline $90 \%$ SC interval & $(2448,2656)$ & $(34,38)$ \\
\hline 95\% SC interval & $(2426,2678)$ & $(33,39)$ \\
\hline * rounded values
\end{tabular}

As far as the mean delay is concerned, Table 2 and Table 3 also show the results of the analysis carried out by the authors in order to estimate the Statistical
Confidence (SC) limits and the associate probability $(90 \%$ and $95 \%)$. Being the number of the observed delays equal to 764 and 1038 for Case A and Case B respectively, the authors have assumed that, according to the central limit theorem, the means of the sample are normally distributed.

At last, Figures 7 and 8 show the behaviour of the delay Cumulative Distribution Functions for the whole set of the observed trains (Case A and Case B respectively). In particular, the curves represent, given a generic time $t$, the probability that the delay is less than $t$ and so furnish the estimate of the Service Dependability of the system. In Figures 7 and 8, the curves characterised by a continuous line have been directly derived from the simulated samples, while the curves characterised by a dashed line, presented just as an example, have been plotted by approximating the delay histogram with an exponential distribution, through the mean values of Tables 2 and 3 respectively. Although no best fitting analysis has been carried out by the authors, such rough and preliminary approximation appears quite acceptable for the simulated case studies.

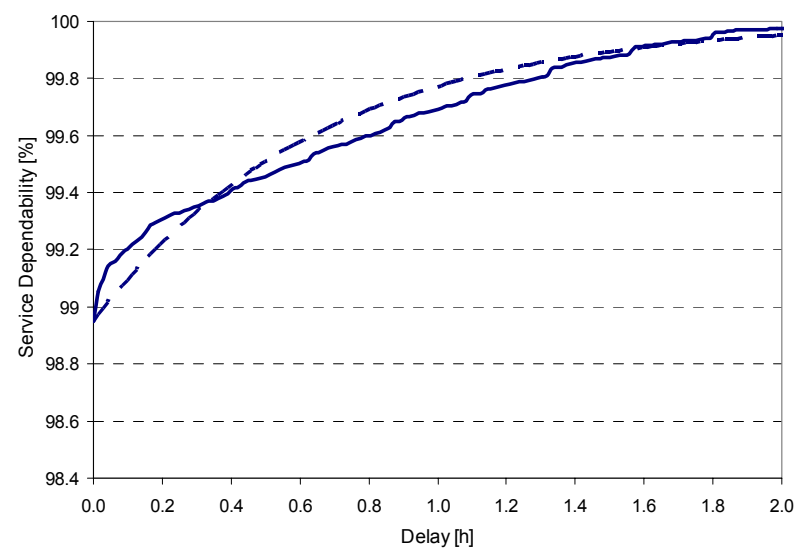

Figure 7 - Service Dependability (Case A)

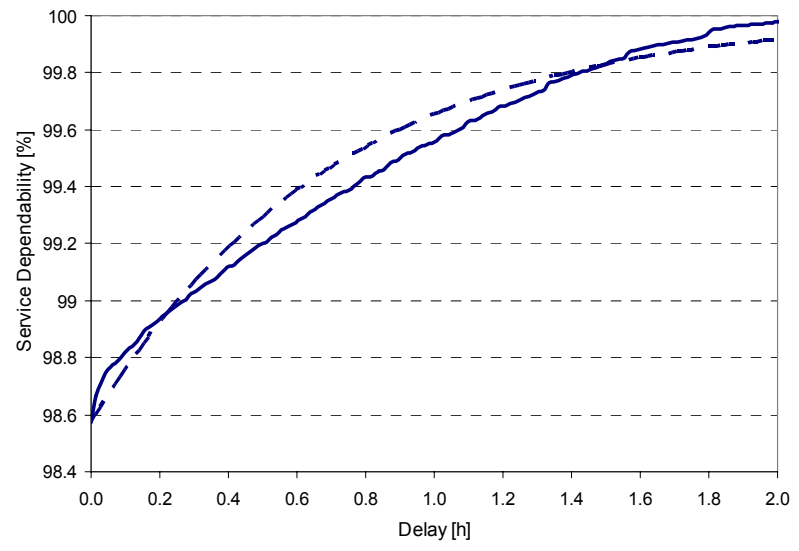

Figure 8 - Service Dependability (Case B)

Stated that the adopted fleet size revealed sufficient to avoid lost runs or delayed departures, that is to say the observed delays are just caused by items failure, it is important to remark the impact of maintenance policies on the service quality. By comparing the results related 
to the whole set of runs, it turns out a not negligible increase of the train mean delay and a decrease of the Service Dependability for Case B, due to the reduction of the frequency of preventive maintenance operations on the mechanical equipment.

\section{CONCLUSIONS}

The results of the impact analysis presented in this paper clearly show the effectiveness of the procedure proposed and based on the adoption of an innovative simulation tool developed by the authors. Such a procedure allows to effectively analyse and solve different problems concerning railway and metro systems. In particular, the main function the tool has been designed for is to provide the user with a set of indicators related to the quality of the service in real operating conditions, and able to support the choice of the optimal solution (as far as, for instance, traffic management rules, maintenance strategies and fleet consist are concerned) during the design phase of a railway or metro system or the choice of the most effective corrective actions to be implemented, when required, in an existing system. Such support is realized by providing an estimate of the impact on the system performances of components failures, traffic management policies, fleet management and maintenance strategies. The added value of such an impact estimate is twofold: on one hand it is customer oriented, as it allows to identify the influence of a particular choice on the quality perceived by the final users (passengers), on the other hand it is decision maker oriented, as it provides all the information to achieve the needed quality targets taking under control the life cycle cost of the system.

\section{REFERENCES}

Bertini, S.; T. Ghiara; and M. Marchesoni. 1990. "AC/DC/AC High voltage traction drives with quasi-zero reactive power demand". Proceedings 6th Int. Conf. PEMC '90, Budapest, Hungary, 1-3 October.

Bogliani, P.; A. Colombo; A. Farini; and F. Fugo. 1992. "Il Pendolino Politensione: le motivazioni della innovazione". Proceedings Conf. Sviluppo e prospettive dei trasporti elettrificati: ricerca ed innovazione, Genova, Italy, 25-27 November, Vol. 1, 265-274.

Bozzo, R.; M. Marchesoni; and S. Savio. 2003. "The impact of power electronics reliability on train delay for conventional and innovative AC traction electrical drives". Proceedings 10th European Conference on Power Electronics and applications EPE '2003, Toulouse, France, 2-4 September.

Calabria, R.; L. Della Ragione; G. Pulcini; and M. Rapone. 1993. "Service Dependability of Transit Systems: a Case Study". Proceedings of Annual Reliability and Maintainability Symposium, Atlanta, Georgia 26-28 January, 366-371.

Cosulich, G.; P. Firpo; and S. Savio. 1996. "Power electronics reliability impact on service dependability for railway systems: a real case study". Proceedings IEEE Int. Conf. on Industrial Electronics ISIE '96, Warsaw, Poland, 17-20 June, 996-1001.
Dazzi, G.; P. Firpo; and S. Savio. 2006. "A modular tool for Service Dependability estimate in railway and metro systems". Proceedings International Symposium of Transport Simulation ISTS 2006, Lausanne, Switzerland, 4-6 September.

Gedam S.G. and S.T. Beaudet. 2000. "Monte Carlo Simulation using Excel $^{\circledR}$ Spreadsheet for Predicting Reliability of a Complex System". Proceedings of Annual Reliability and Maintainability Symposium, Los Angeles, California, USA, 24-27 January, 188-193.

IEEE Std 493. 1990. Recommended Practice for the Design of Reliable Industrial and Commercial Power Systems. IEEE Standards Department, Piscataway, New Jersey, USA.

MIL-HDBK-217. 1991. Reliability Prediction of Electronic Equipment. U.S. Department of Defense, Washington DC, USA.

\section{AUTHOR BIOGRAPHIES}

GIOVANNI DAZZI graduated in Mechanical Engineering at the University of Genoa in 2004 and is currently attending a $\mathrm{PhD}$ course in Electrical Engineering at the Electrical Engineering Department of the same University. His research activities deal with the RAMS analysis and modelling of electrical and transportation systems.

PIERLUIGI FIRPO graduated in Electronic Engineering at the University of Genova, Italy, in 1991, received his Ph.D. in Electrical Engineering from the same University in 1994 and is currently working at D’Appolonia S.p.A. as Manager of the Railway Systems and Technologies Business Unit. His main interests are in the field of Railway and Metrorail Systems Engineering with special focus on safety and certification aspects. He also worked for the company Sciro S.p.A., formerly Sciro Electra, in the field of RAMS and V\&V applied to guided transport systems. He has been covering the role of Managing Director of the certification body SciroTÜV contributing to its appointment as Notified Body (N. 1287) according to the European Directive 96/48/CE on the Interoperability of the Transeuropean High Speed Railway System. He joined D'Appolonia since March 2004. He is Member of IEEE since 1994 and is author of about fifty publications.

STEFANO SAVIO received the Ph.D. degree in Electrical Engineering from the University of Genova, Italy, in 1989. Associate Professor at the Electrical Engineering Department of Genova, he is author of more than 100 papers, most of them dealing with the Product Assurance assessment for industrial and transportation systems, as far as modelling and simulation oriented to RAMS prediction are concerned. As far as applied research is concerned, he was Evaluation Manager of the EU projects MARCO and COMBINE, Project Coordinator of the EU projects COMBINE 2 and F-MAN, and is currently Leader of the Work Package Reliability and Economics within the EU project UNIFLEX-PM. 